\title{
Analytic Approaches for the Transient Temperature Distribution in a Single Cable for Smart Fuses and Ampacity Derating Calculation
}

\author{
Anika Henke, Stephan Frei \\ On-board Systems Lab, TU Dortmund University \\ Otto-Hahn-Str. 4, 44227 Dortmund, Germany \\ anika.henke@tu-dortmund.de
}

\begin{abstract}
To protect cables from damage by too high temperatures, classically fuses are used. Those often lead to over-dimensioned cables due to their melting behaviour or simple tripping strategies. Therefore, smart fuses can be advantageous. Here, a more powerful control circuit can estimate the cable temperature and operate as switch, when the temperature exceeds a given limit. The control circuit needs to run a thermal simulation of the cable to be protected. Critical temperatures can be detected this way. Thermal cable models can be very complex and computationally intensive, especially when numerical methods are used. In this contribution, a new analytic approach for the transient axial temperature distribution along a single cable is presented. Using this solution, a significant reduction of the time needed for calculation is achieved.
\end{abstract}

Keywords: Analytic calculation, Laplace transform, Thermoelectric equivalent, Partial differential equation, Single cable, Thermal modelling

\section{Introduction}

Driven by the current developments concerning electric mobility and an increasing number of electronic devices, higher voltages and currents appear in automobiles. Cables to carry the high currents need to be chosen carefully: On the one hand, the smaller the diameter of the cables is, the lower the weight and the higher the flexibility is. On the other hand, a smaller diameter of the cable leads to a higher resulting temperature. The temperature highly affects the aging of the cable isolation and therefore the lifetime. To avoid temperature damages, classical melting fuses can be used. Those trigger dependent on the temperature of the melting wire and cannot consider the actual condition of the cable that should be protected. Worst-case conditions have to be assumed, which might cause over-dimensioned cables. In addition, melting fuses have to be exchanged after triggering once, so all of the fuses have to be accessible easily. To avoid these disadvantages, electronic and smart fuses were developed, requiring methods for overload detection. One approach is based on a model of the cable, which is used to calculate the cable temperature with the measured current as input. If the calculated cable temperature rises above a critical temperature, the fuse interrupts the current with a semiconductor or relay [1]. Therefore, precise and fast models to approximate the temperature in the cable are needed.

The simplest approach is to model only the radial heat flow (see e.g. [2-6]) and neglect the axial heat flow along the cable. For special set-ups, analytic formulas for the temperature can be found (e.g. [7,8]). Only mentioning the radial heat flow is equivalent to describing an infinitely long cable. Therefore, the real temperature in the middle of the cable is only calculated correctly, if the beginning and the end of the cable do not influence the temperature in the middle of the cable. This means that sufficiently long cables in a uniform environment have to be assumed. Unlike, in real structures, often the temperatures at the beginning and the end of the cable are relevant for the maximal temperature along the cable. That is why the axial heat flow has to be considered to find realistic temperatures.

Considering the axial heat flow along the cable leads to a significantly higher complexity of the resulting problem. That is why in previous works, axial models based on numerical calculation methods were derived. For example, in [9], equivalent circuits are used. The axial model is build up by connecting several instances of the radial model in series via axial heat transfer resistances in MATLAB/Simscape. So, the time-dependent calculation of the temperature distribution along the line causes a high calculation effort. In [10], differential equations for the stationary case are derived and solved using the finite element method. In [11], a model of a power cable is build up using a 3D finite element method. This is used to evaluate the temperature of the cable. Another approach found in literature as e.g. [12] is to model the axial heat 
flux along the cable, but to take into account only the stationary case without modelling of the time dependency. As transient information is highly relevant for real applications, neglecting the time dependency can severely limit the practical applicability of those models.

In the electrical transmission line theory, axial voltage and current distributions along cables are calculated as solutions of a differential equation, which is derived from an equivalent circuit for an infinitely short cable [13]. Analogous, in this paper, thermoelectric equivalent circuits for an infinitely short segment of a cable are used to derive a differential equation for the temperature along the cable. This approach is only valid for special surrounding conditions as for example a constant ambient temperature and a constant current through the cable. In contrast to the aforementioned numerical approximations, in this contribution, a new analytic approximation for the solution of the resulting partial differential equation for the thermal modelling of a single air-cooled cable is presented. In contrast to previous models, the temperature at each position along the cable and each point in time can be calculated independent from each other, which can reduce the calculation time for concrete applications drastically. This model can be used in smart fuses, but also for the dimensioning of cables. In the following chapter 2, the examined model and the analytic solution are presented. In chapter 3 , some details concerning the implementation are given, before results are shown in chapter 4 .

\section{Derivation of an analytic solution for a single cable}

In this chapter, the theoretical approaches for a new analytic solution are shown. After a brief summary about the modelled configuration and the presentation of the thermoelectric model, the different solutions are derived.

\subsection{Examined model of a single cable}

Fig. 1 shows the modelled cable of length $L$, oriented in $z$-direction. It consists of a solid copper conductor with radius $r_{\mathrm{c}}$ surrounded by an isolation (PVC). The outer radius of the cable is $r_{\mathrm{i}}$. A current $I$ flows through the cable, which is terminated with panels with infinite heat capacitance and temperature $T_{1}$ respectively $T_{2}$ at the beginning respectively end. The ambient air has the temperature $T_{\mathrm{E}}$. The coordinate $z$ is zero at the left termination.

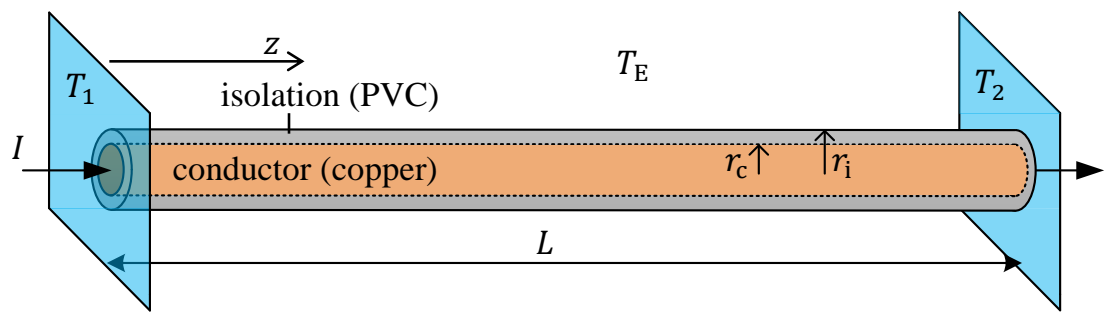

Fig. 1: Modelled cable in the assumed environment.

\subsection{Fundamental thermoelectric model for a single transmission cable}

The cable is modelled with a thermoelectric equivalent circuit: Analogous to the electric domain, an equivalent circuit is derived. In analogy to electric transmission line theory [13], the partial differential equation is found by consideration of an infinitely short segment of the cable. The cable parameters are all given related to length as per unit length parameters (pul) and marked with an upstroke, e.g. $P_{\mathrm{e}}^{\prime}, C^{\prime}, R_{\mathrm{L}}{ }^{\prime}$. To reduce the complexity of the model, some assumptions are made: At first, the thermal conductivity of the conductor is assumed to be very high, so the temperature gradient in radial direction along the conductor is neglected and only the temperature gradient along the isolation remains in radial direction. Therefore, the isolation has a quite low thermal conductivity. This is also the reason why in axial direction, the influence of the isolation is neglected, so only the influence of the conductor remains. The resulting equivalent circuit of an infinitesimal short cable segment is shown in fig. 2(a). The following descriptions concerning the parameters are based on [9]. 

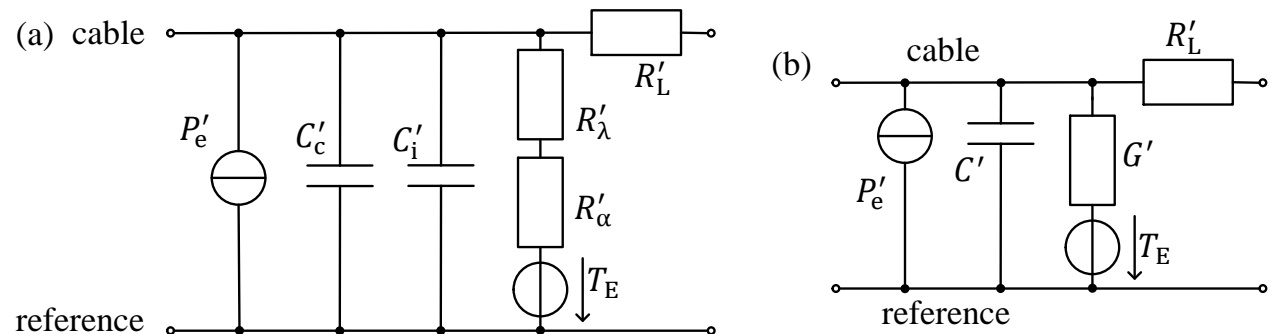

Fig. 2: Thermoelectric equivalent model to describe the heat flow through the cable. (a) Detailed model to describe physical effects.

(b) Condensed model.

An overview of the formulas for the used radial parameters is given in the following Table 1 . The heat that is injected into the system via the source $P_{\mathrm{e}}^{\prime}$ represents the pul electrical losses because of the electrical current $I$ in the cable. The linear temperature coefficient for conductor conductivity is $\alpha_{\mathrm{T}} . R_{\text {ref }}^{\prime}$ means the electric pul resistance at the temperature $T_{\text {ref }}$ and $T$ means the conductor temperature. The thermal pul capacitances for the conductor $C_{\mathrm{c}}^{\prime}$ and the isolation $C_{\mathrm{i}}^{\prime}$ depend on the specific heat capacities per volume $c_{\mathrm{c}}$ and $c_{\mathrm{i}}$. The thermal pul resistance $R_{\lambda}^{\prime}$ describes the thermal heat flow through the isolation with the thermal conductivity of the isolation $\lambda_{\mathrm{i}}$. The thermal pul resistance $R_{\alpha}^{\prime}$ describes the heat transfer from the isolation to the surrounding air with convection and radiation. The heat transfer coefficient $\alpha_{\mathrm{r}}$ describes the radiation. The temperatures $T_{\mathrm{S}, \mathrm{K}}$ and $T_{\mathrm{E}, \mathrm{K}}$ are the temperature of the surface of the isolation respectively the environmental temperature in Kelvin, $\varepsilon$ is the emissivity of the isolation material and $\sigma \approx 5.6704 \cdot 10^{-8} \mathrm{~W} / \mathrm{m}^{2} \mathrm{~K}^{4}$ is the StefanBoltzmann constant. As in [9], the emissivity is assumed to be 0.95 in this paper. The heat transfer coefficient $\alpha_{\mathrm{k}}$ describes free convection in air. $\lambda_{\text {Air }}$ means the thermal conductivity of air and $l_{\alpha}=2 r_{\mathrm{i}}$ the characteristic length of the structure. The Nusselt number $\mathrm{Nu}\left(T_{\mathrm{S}}, T_{\mathrm{E}}\right)$ [14] is depending on the structure: For a cylinder it is calculated using the Rayleigh number $\mathrm{Ra}=\mathrm{Gr} \cdot \mathrm{Pr}$, the Grashof number Gr and the Prandtl number $\mathrm{Pr}$, with the gravity of the earth $g \approx 9.81 \mathrm{~m} / \mathrm{s}^{2}$, the coefficient of thermal expansion $\beta=1 / T_{\mathrm{E}, \mathrm{K}}$ and the kinematic viscosity $v$ [15]. The parameters $\lambda_{\text {Air }}, v$ and $\operatorname{Pr}$ can be determined from tables as for example given in [14] using the mean temperature $T_{\mathrm{m}}=0.5\left(T_{\mathrm{s}}+T_{\mathrm{E}}\right)$, so they depend on the actual surface temperature of the cable $T_{\mathrm{s}}$. If $T_{\mathrm{m}}$ is in between the values mentioned in [14], a spline interpolation between the given values is used. All of the aforementioned parameters describe the radial heat flow in the cable. For the axial behaviour, the axial thermal resistance per length $R_{\mathrm{L}}^{\prime}$ is introduced with the thermal conductivity of the conductor $\lambda_{\mathrm{c}}$. For the calculations, the model is condensed to the form presented in fig. 2(b). The new parameters $C^{\prime}$ and $G^{\prime}$ are calculated from the already presented parameters.

Table 1: Overview of the calculation rules for the radial parameters.

\begin{tabular}{|c|c|c|c|c|}
\hline meaning & \multicolumn{2}{|l|}{ calculation rule } & meaning & calculation rule \\
\hline electrical losses & \multicolumn{2}{|c|}{$P_{\mathrm{e}}^{\prime}=I^{2} R_{\mathrm{ref}}^{\prime}\left(1+\alpha_{\mathrm{T}}\left(T-T_{\mathrm{ref}}\right)\right)$} & free convection & $\alpha_{\mathrm{k}}=\mathrm{Nu}\left(T_{\mathrm{s}}, T_{\mathrm{E}}\right) \cdot \lambda_{\mathrm{Air}} / l_{\alpha}$ \\
\hline $\begin{array}{l}\text { heat transfer (isolation } \\
\text { to surrounding air) }\end{array}$ & \multicolumn{2}{|c|}{$R_{\alpha}^{\prime}=1 /\left[\left(\alpha_{\mathrm{k}}+\alpha_{\mathrm{r}}\right) 2 \pi r_{\mathrm{i}}\right]$} & $\begin{array}{r}\text { thermal capacitance } \\
\text { (conductor) }\end{array}$ & $C_{\mathrm{c}}^{\prime}=c_{\mathrm{c}} \pi r_{\mathrm{c}}^{2}$ \\
\hline radiation & \multicolumn{2}{|c|}{$\alpha_{\mathrm{r}}=\varepsilon \cdot \sigma \cdot\left(T_{\mathrm{s}, \mathrm{K}}+T_{\mathrm{E}, \mathrm{K}}\right) \cdot\left(T_{\mathrm{s}, \mathrm{K}}^{2}+T_{\mathrm{E}, \mathrm{K}}^{2}\right)$} & $\begin{array}{r}\text { thermal capacitance } \\
\text { (isolation) }\end{array}$ & $C_{\mathrm{i}}^{\prime}=c_{\mathrm{i}} \pi\left(r_{\mathrm{i}}^{2}-r_{\mathrm{c}}^{2}\right)$ \\
\hline \multirow{4}{*}{ Nusselt number } & \multirow{4}{*}{$\mathrm{Nu}\left(T_{\mathrm{s}}, T_{\mathrm{E}}\right)=$} & \multirow[b]{2}{*}{$0,387 \cdot \mathrm{R}^{\frac{1}{6}}$} & $\begin{array}{l}\text { thermal heat conduc- } \\
\text { tion through isolation }\end{array}$ & $R_{\lambda}^{\prime}=\ln \left(\frac{r_{\mathrm{i}}}{r_{\mathrm{c}}}\right) /\left(2 \pi \lambda_{\mathrm{i}}\right)$ \\
\hline & & & electric resistance & $R_{\mathrm{ref}}^{\prime}=\rho /\left(\pi \cdot r_{\mathrm{c}}^{2}\right)$ \\
\hline & & $\left(0559 \frac{9}{16}\right)^{\frac{8}{27}}$ & axial thermal resistance & $R_{\mathrm{L}}^{\prime}=1 /\left(\lambda_{\mathrm{c}} \cdot \pi \cdot r_{\mathrm{c}}^{2}\right)$ \\
\hline & & $1+\left(\frac{\operatorname{Pr}}{\operatorname{Pr}}\right)$ & \multirow{2}{*}{$\begin{array}{l}\text { new parameters for } \\
\text { condensed model }\end{array}$} & $C^{\prime}=C_{\mathrm{c}}^{\prime}+C_{\mathrm{i}}^{\prime}$ \\
\hline Grashof number & $\mathrm{Gr}=g \cdot l_{\alpha}^{3} \cdot \beta$ & $\left|T_{\mathrm{s}}-T_{\mathrm{E}}\right| / v^{2}$ & & $G^{\prime}=1 /\left(R_{\lambda}^{\prime}+R_{\alpha}^{\prime}\right)$ \\
\hline
\end{tabular}


In the next step, the differential equation is deduced from the circuit model. Like in the electric domain, Kirchhoff's laws are evaluated. Rearranging of the resulting formulas leads to the inhomogeneous partial differential equation, which describes the temperature distribution along the cable:

$$
\frac{1}{R_{\mathrm{L}}^{\prime}} \frac{\mathrm{d}^{2} T(z, t)}{\mathrm{d} z^{2}}-G^{\prime} T(z, t)-C^{\prime} \frac{\mathrm{d} T(z, t)}{\mathrm{d} t}=P_{\mathrm{e}}^{\prime}-G^{\prime} T_{\mathrm{E}}
$$

The elements $G^{\prime}$ and $P_{\mathrm{e}}^{\prime}$ of the circuit, that lead to the parameters of the differential equation, are dependent of the temperature. That is why the differential equation is nonlinear. This dependency is very complicated, so for this moment it is neglected. Subsequently, the dependency is included by finding a self-consistent solution using a few iterations.

\subsection{Stationary solution}

At first, a stationary solution of the differential equation (1) is presented. In the stationary case, there is no variation of the temperature along the time. In the reduced differential equation, only the variable $z$ remains. The solution includes a homogenous and a particulate term. The constant factors $T_{\mathrm{h} 1}$ and $T_{\mathrm{h} 2}$ follow from the initial conditions $T(0)=T_{1}$ and $T(L)=T_{2}$ (temperature at the beginning and the end of the cable):

$$
\begin{gathered}
T(z)=T_{\mathrm{h} 1} \exp \left(\sqrt{R_{\mathrm{L}}^{\prime} G^{\prime} z}\right)+T_{\mathrm{h} 2} \exp \left(-\sqrt{R_{\mathrm{L}}^{\prime} G^{\prime} z}\right)-\frac{P_{\mathrm{e}}^{\prime}}{G^{\prime}}+T_{\mathrm{E}}, \\
T_{\mathrm{h} 2}=\frac{T_{2}-T_{\mathrm{E}}+\frac{P_{\mathrm{e}}^{\prime}}{G^{\prime}}-\left(T_{1}-T_{\mathrm{E}}+\frac{P_{\mathrm{e}}^{\prime}}{G^{\prime}}\right) \exp \left(\sqrt{R_{\mathrm{L}}^{\prime} G^{\prime}} L\right)}{\exp \left(-\sqrt{R_{\mathrm{L}}^{\prime} G^{\prime}} L\right)-\exp \left(\sqrt{R_{\mathrm{L}}^{\prime} G^{\prime}} L\right)}, \quad T_{\mathrm{h} 1}=T_{1}-T_{\mathrm{E}}+\frac{P_{\mathrm{e}}^{\prime}}{G^{\prime}}-T_{\mathrm{h} 2 .}
\end{gathered}
$$

\subsection{Complete solution for a single cable}

The differential equation (1) is now solved using the Laplace transform. The temperature of the conductor at $t=0 \mathrm{~s}$ is $T(z, 0)=T_{0}=$ const.. The temperature at the beginning of the conductor is $T(0, t)=T_{1}$ and at the end of the conductor $T(L, t)=T_{2}$ for all times. The Laplace transform yields

$$
\frac{1}{R_{\mathrm{L}}^{\prime}} \frac{\mathrm{d}^{2} T(z, s)}{\mathrm{d} z^{2}}-\left(G^{\prime}+s C^{\prime}\right) T(z, s)=\frac{P_{\mathrm{e}}^{\prime}-G^{\prime} T_{\mathrm{E}}}{s}-C^{\prime} T_{0}, \quad T(0, s)=\frac{T_{1}}{s}, \quad T(L, s)=\frac{T_{2}}{s}
$$

The remaining differential equation only depends on the variable $z$ and can be solved as before. The factors $A_{1}(s)$ and $A_{2}(s)$ are again found using the remaining boundary conditions.

$$
\begin{gathered}
T(z, s)=A_{1}(s) \exp \left(z \sqrt{R_{\mathrm{L}}^{\prime}\left(G^{\prime}+C^{\prime} s\right)}\right)+A_{2}(s) \exp \left(-z \sqrt{R_{\mathrm{L}}^{\prime}\left(G^{\prime}+C^{\prime} s\right)}\right)-\frac{P_{\mathrm{e}}^{\prime}-G^{\prime} T_{\mathrm{E}}-s C^{\prime} T_{0}}{s\left(G^{\prime}+s C^{\prime}\right)}, \\
A_{1}(s)=\frac{T_{1}}{s}+\frac{P_{\mathrm{e}}^{\prime}-G^{\prime} T_{\mathrm{E}}-s C^{\prime} T_{0}}{s\left(G^{\prime}+s C^{\prime}\right)}-A_{2}(s), A_{2}(s)=\frac{\frac{T_{2}}{s}+\frac{P_{\mathrm{e}}^{\prime}-G^{\prime} T_{\mathrm{E}}-s C^{\prime} T_{0}}{s\left(G^{\prime}+s C^{\prime}\right)}-\left(\frac{T_{1}}{s}+\frac{P_{\mathrm{e}}^{\prime}-G^{\prime} T_{\mathrm{E}}-s C^{\prime} T_{0}}{s\left(G^{\prime}+s C^{\prime}\right)}\right) \exp \left(\sqrt{R_{\mathrm{L}}^{\prime}\left(G^{\prime}+C^{\prime} s\right)} L\right)}{\exp \left(-\sqrt{R_{\mathrm{L}}^{\prime}\left(G^{\prime}+C^{\prime} s\right)} L\right)-\exp \left(\sqrt{R_{\mathrm{L}}^{\prime}\left(G^{\prime}+C^{\prime} s\right)} L\right)}
\end{gathered}
$$

In the next step, the solution in the Laplace domain has to be transformed back into the time domain. As numeric inverse Laplace transformation algorithms should be avoided, an analytic approach has to be found. There are different terms in the Laplace solution, of which not all have a known transformation back into the time domain: The terms in which

the exponential function appears in the numerator as well as in the denominator lead to complications. That is why $A_{1}(s)$ 
and $A_{2}(s)$ are approximated in the next step: By inserting typical values, it is shown, that $\exp \left(-L \sqrt{R_{\mathrm{L}}^{\prime}\left(G^{\prime}+C^{\prime} s\right)}\right)$ can be neglected against \pm 1 . This leads to the reduced form for the factors:

$$
A_{2} \approx \frac{P_{\mathrm{e}}^{\prime}-G^{\prime} T_{\mathrm{E}}-s C^{\prime} T_{0}}{s\left(G^{\prime}+s C^{\prime}\right)}+\frac{T_{1}}{s}, \quad A_{1} \approx\left(\frac{P_{\mathrm{e}}^{\prime}-G^{\prime} T_{\mathrm{E}}-s C^{\prime} T_{0}}{s\left(G^{\prime}+s C^{\prime}\right)}+\frac{T_{2}}{s}\right) \exp \left(-\sqrt{R_{\mathrm{L}}^{\prime}\left(G^{\prime}+C^{\prime} s\right)} L\right)
$$

The approximated result in the Laplace domain can be transformed back into the time domain. The complete process of the solution including the result in the time domain is presented in fig. 3.

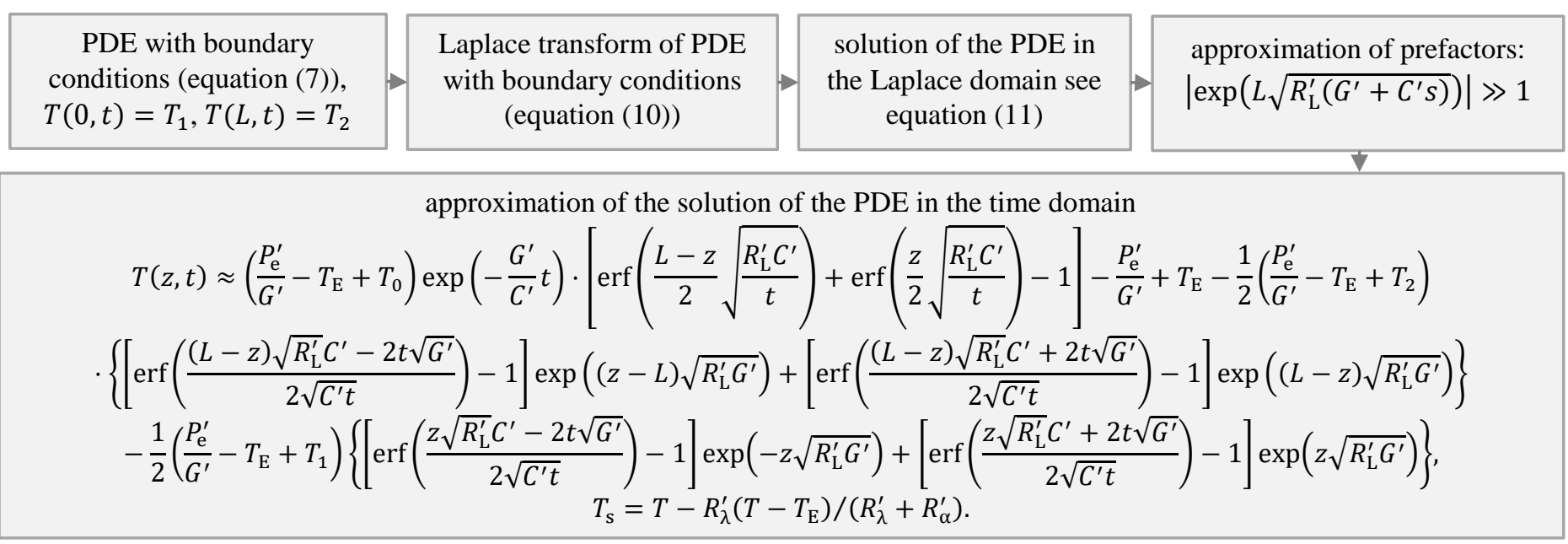

Fig. 3: Process to find the approximation of the solution of the partial differential equation.

\subsubsection{Infinitely long cable}

For an infinitely long cable, only the particulate term remains, which can be transformed back into the time domain easily. As expected, the $z$-dependency vanishes.

$$
T(z, s)=-\frac{P_{\mathrm{e}}^{\prime}-G^{\prime} T_{\mathrm{E}}-s C^{\prime} T_{0}}{s\left(G^{\prime}+s C^{\prime}\right)} \Rightarrow T(z, t)=-\frac{P_{\mathrm{e}}^{\prime}-G^{\prime} T_{\mathrm{E}}}{G^{\prime}}\left(1-\exp \left(-\frac{G^{\prime}}{C^{\prime}} t\right)\right)+T_{0} \exp \left(-\frac{G^{\prime}}{C^{\prime}} t\right)
$$

\subsubsection{Half infinite cable}

In this section, a cable is modelled, which starts at $z=0 \mathrm{~m}$ and is of infinite length. Because of the infinite length of the cable, the factor $A_{1}(s)$ has to vanish: $A_{1}(s)=0$. The other factor is calculated from the remaining boundary condition $T(0, t)=T_{1}=$ const.. The inverse Laplace transform leads to the solution in the time domain.

$$
\begin{gathered}
T(z, t)=\frac{1}{2}\left\{\left[1-\operatorname{erf}\left(\frac{z \sqrt{R_{\mathrm{L}}^{\prime}} C^{\prime}-2 \sqrt{G^{\prime}} t}{2 \sqrt{C^{\prime}} \sqrt{t}}\right)\right] \exp \left(-z \sqrt{R_{\mathrm{L}}^{\prime} G^{\prime}}\right)+\left[1-\operatorname{erf}\left(\frac{z \sqrt{R_{\mathrm{L}}^{\prime}} C^{\prime}+2 \sqrt{G^{\prime}} t}{2 \sqrt{C^{\prime}} \sqrt{t}}\right)\right] \exp \left(z \sqrt{R_{\mathrm{L}}^{\prime} G^{\prime}}\right)\right\} \\
\cdot\left(T_{1}-T_{\mathrm{E}}+\frac{P_{\mathrm{e}}^{\prime}}{G^{\prime}}\right)+\operatorname{erf}\left(\frac{z \sqrt{R_{\mathrm{L}}^{\prime} C^{\prime}}}{2 \sqrt{t}}\right) \exp \left(-\frac{G^{\prime}}{C^{\prime}} t\right)\left(T_{0}-T_{\mathrm{E}}+\frac{P_{\mathrm{e}}^{\prime}}{G^{\prime}}\right)-\frac{P_{\mathrm{e}}^{\prime}}{G^{\prime}}+T_{\mathrm{E}}
\end{gathered}
$$

\section{Implementation of the developed solutions}

In all of the before presented solutions, constant values for the parameters in the equivalent circuit are assumed. As one can see from the calculation rules presented in chapter 2.2, the parameters are dependent on the temperatures of the 
conductor and the surface of the isolation. The surface temperature of the isolation is dependent of the temperature of the conductor and again of the parameters. The temperature of the conductor is calculated using known parameters. Those relations are shown in fig. 4(a). All in all, a self-consistent problem results. That is why an iterative approach is used here (see fig. 4(b)): Starting with an estimated temperature for the conductor and typical values for the parameters, the corresponding temperature of the surface of the isolation is calculated. Using this, the parameters are recalculated. In the last step of the first iteration, the temperature of the conductor is calculated. With this new temperature, the temperature of the surface is recalculated and so on. This process can be repeated a few times to find a solution, in which parameters and the mentioned temperatures fit together. After a few iterations (maximal 10 for the examined cases) only minor corrections $(\ll 1 \mathrm{~K})$ result from further iterations. Generally, for every time and every position along the cable, the iterations have to be done again. This means quite some effort, if a very dense mesh of time and space points should be calculated. But there is a huge advantage: The temperature at each point in time and space is calculated absolutely independent from each other point, which means, that neither the whole cable has to be calculated if only a special point is of interest nor earlier time steps need to be calculated if only a later point is relevant. Compared to the numeric calculation of the solution of the differential equation this leads to an enormous reduction of the computation time.

In the beginning, the stationary solution is calculated. For the calculation of the infinite cable, the stationary solution in the middle of the cable is used as first guess for the temperature of the conductor. The complete solution as well as the half infinite solution are dependent of the time and the position along the cable. For the half infinite solution, the stationary temperature is used as first guess for the conductor temperature of the first half of the cable, for the second half the stationary solution in the middle of the cable is used. For the complete solution, the stationary solution at the specific point of the cable is used. For each desired time the iterations are repeated until convergence is reached. As mentioned before, for the examined cases maximal 10 iterations are necessary. The complete process is summed up in fig. 4(c).

(a)

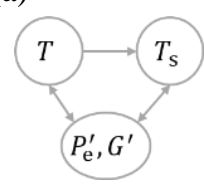

(b)

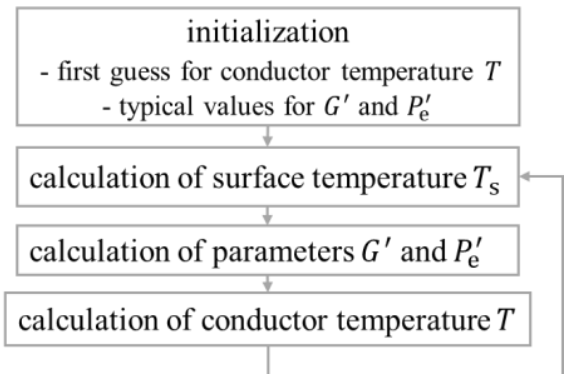

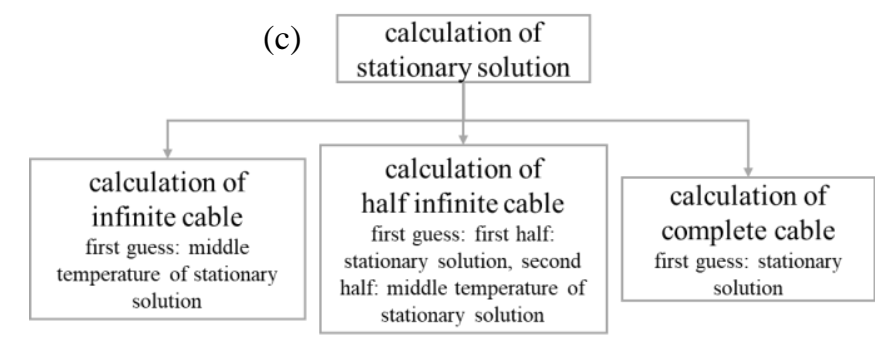

Fig. 4: (a) Dependency between the temperature of the conductor $T$ and the surface $T_{\mathrm{s}}$ of the cable and the parameters $P_{\mathrm{e}}^{\prime}, G^{\prime}$. (b) Scheme of the iterations to include nonlinear parameters. (c) Usage of the stationary solution as first guess for other calculations.

\section{Numeric reference solution of the partial differential equation}

The numeric solution of the differential equation presented in equation (1) is used to validate the analytical approximation. The numeric solution is implemented applying the Crank-Nicholson method and three iteration steps (see previous chapter), so these iteration steps consider the nonlinear terms. This solution will be regarded as correct and used as reference solution for the following comparisons. A discretization of 100 steps for the $z$-direction and 1001 steps for the time is used.

\section{Results}

The presented solutions are examined for the cable presented in fig. 1: A current of 70 A flows through a $6 \mathrm{~mm}^{2}$ - cable (radius of the conductor: $r_{\mathrm{c}}=1.382 \mathrm{~mm}$, total radius with isolation $r_{\mathrm{i}}=2 \mathrm{~mm}$ ) with a length of $1 \mathrm{~m}$. The surrounding air has a temperature of $T_{\mathrm{E}}=25{ }^{\circ} \mathrm{C}$, which is as well the temperature of the cable at $t=0 \mathrm{~s}\left(T_{0}\right)$ and of the beginning $\left(T_{1}\right)$ and the end $\left(T_{2}\right)$ of the cable for all times. The conductor consists of solid copper (specific heat capacity $c_{\mathrm{c}}=3.4 \cdot 10^{6} \mathrm{~J} / \mathrm{m}^{3} \mathrm{~K}$, thermal conductivity $\lambda_{\mathrm{c}}=386 \mathrm{~W} / \mathrm{Km}$, resistivity at $20^{\circ} \mathrm{C}: \rho=1.86 \cdot 10^{-8} \Omega \mathrm{m}$ ) and the isolation of PVC (specific heat 
capacity $c_{\mathrm{i}}=2.245 \cdot 10^{6} \mathrm{~J} / \mathrm{m}^{3} \mathrm{~K}$, thermal conductivity $\lambda_{\mathrm{i}}=0.21 \mathrm{~W} / \mathrm{Km}$, emissivity $\left.\varepsilon=0,95\right)$. The temperature coefficient is $\alpha_{\mathrm{T}}=3.93 \cdot 10^{-3} 1 / \mathrm{K}$. The analytic approximation for the temperature along the cable is compared to the numeric inversion of the original solution in the Laplace domain to see if the approximation causes errors. The numeric inversion of the Laplace transform is done using the Gaver-Stehfest-algorithm [16] with parameter $M=5$. The results are shown in fig. 5. The good agreement (the results differ in the fourth decimal digit) between both solutions confirms that the approximation is quite exact.
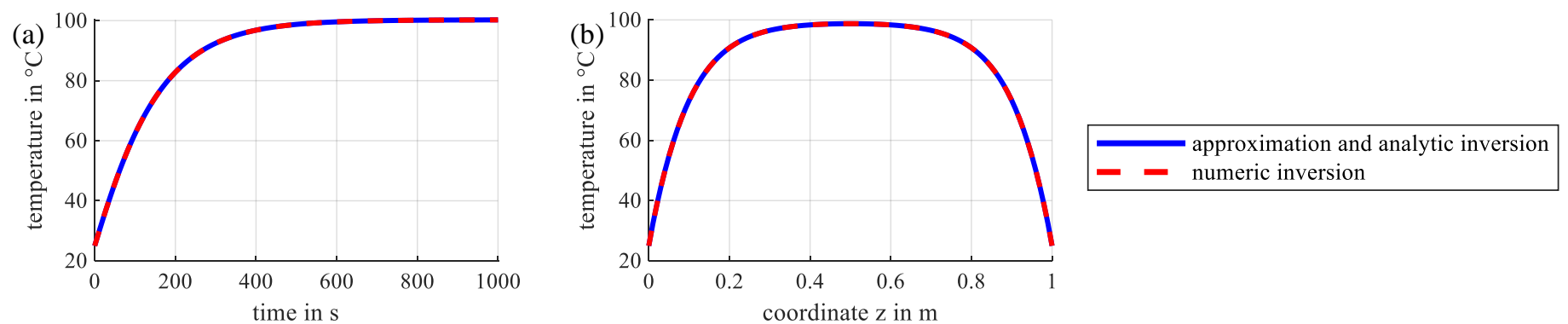

Fig. 5: Comparison of the solution using the numeric inverse Laplace transform and of the approximation with analytic solution. (a) Maximum temperatures of the conductor along time. (b) Temperature of the conductor along the cable at $t=500 \mathrm{~s}$.
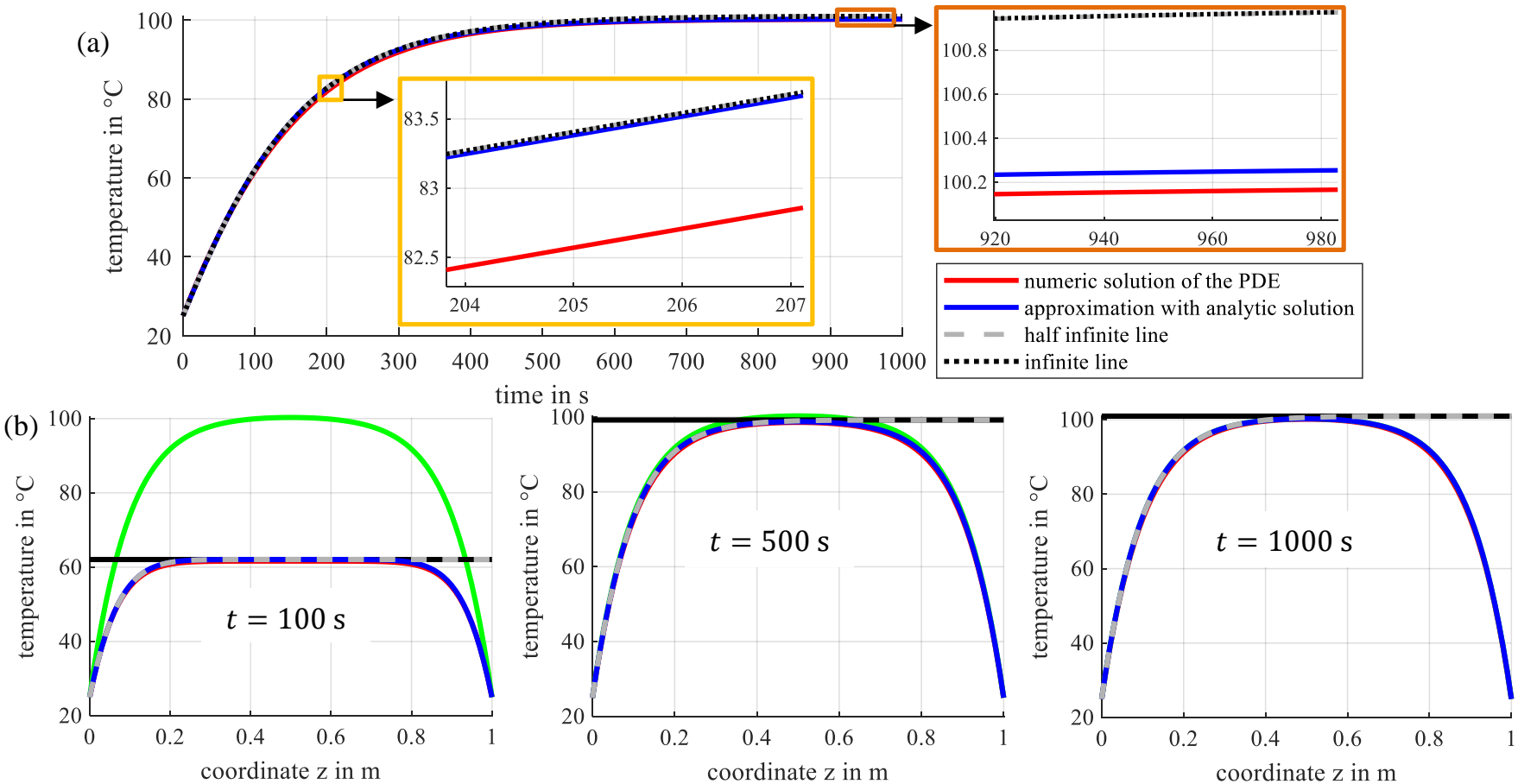
- half infinite line coordinate $\mathrm{Z}$ in $\mathrm{m}$ coordinate $\mathrm{z}$ in $\mathrm{m}$

Fig. 6: (a) Comparison of the maximum temperatures of the conductor along time. Development for $t=0 \mathrm{~s}$ to $t=1000 \mathrm{~s}$ with zoom to special areas. (b) Calculated temperature along the cable for different time steps: $t=100 \mathrm{~s}, t=500 \mathrm{~s}, t=1000 \mathrm{~s}$.

In fig. 6(a), the results for the maximum of the temperature of the conductor over time are shown. The temperature is taken at the middle of the cable. All analytic solutions show a similar development. For long times, the infinite and half infinite solutions show minimal higher values than the complete solution. This is because of the length of the cable: For a longer cable, the values come closer together. The numeric solution shows a different behaviour in the transient state: Up 
to approximately $2 \mathrm{~K}$ lower temperatures are predicted here. This is because in the numeric solution, the results of the iterations propagate to the next time step. As mentioned before, in the analytic solution, each position in time and location is independent from each other position. Another function is used for each step and there is no coupling between the different positions. In the used functions, it is implicitly assumed, that all parameters are constant. That is why especially for the transient case some deviations appear. Nevertheless, the predicted temperature only differs little from the numerically computed temperature and therefore might be used as a good, very fast approximation. In fig. 6(b), the calculated temperature along the cable is shown for different time steps. For long times, the solutions come closer together and meet the stationary solution. In the transient area, the numeric and analytic approaches slightly deviate from each other mainly in the middle of the cable.

\section{Conclusion}

In this contribution, a new analytic approach for the transient axial temperature distribution along a single cable with constant ambient temperature and fixed temperatures at the beginning and the end of the cable is presented. The cable is assumed to have a constant temperature at the beginning. Using an iterative approach, non-linear material parameters can be considered. Compared to known numeric solutions for this configuration, a significant reduction of the calculation time is achieved. The solution is very close to the numeric solution, so it can be used for example in smart fuses. Furthermore,

solutions for cables of infinite length are presented. The usage of the half infinite cable reduces the effort of the computation even further and might be used when accuracy can be lower and computational resources are reduced.

\section{References}

[1] S. Önal and S. Frei, "A model-based automotive smart fuse approach considering environmental conditions and insulation aging for higher current load limits and short-term overload operations," in IEEE International Conference on Electrical Systems for Aircraft, Railway, Ship Propulsion and Road Vehicles \& International Transportation Electrification Conference (ESARS-ITEC), Nottingham, United Kingdom, 2018.

[2] R. Olsen, G. J. Anders, J. Holboell and U. S. Gudmundsdóttir, "Modelling of Dynamic Transmission Cable Temperature Considering Soil-Specific Heat, Thermal Resistivity, and Precipitation," in IEEE Transactions on Power Delivery, 2013, vol. 28, no. 3, pp. 1909-1917.

[3] C. Holyk, H.-D. Liess, S. Grondel, H. Kanbach and F. Loos, "Simulation and measurement of the steady-state temperature in multi-core cables," in Electric Power Systems Research, 2014, vol. 116, pp. 54-66.

[4] M. Lei, G. Liu, Y. Lai, J. Li, W. Li and Y. Liu, "Study on thermal model of dynamic temperature calculation of single-core cable based on Laplace calculation method," in IEEE International Symposium on Electrical Insulation, San Diego, CA, 2010.

[5] D. Lauria, M. Pagano and C. Petrarca, "Transient Thermal Modelling of HV XLPE Power Cables: matrix approach and experimental validation," in IEEE Power \& Energy Society General Meeting (PESGM), Portland, OR, 2018.

[6] Q. Zhan, J. Ruan, K. Tang, L. Tang, Y. Liu, H. Li and X. Ou, "Real-time calculation of three core cable conductor temperature based on thermal circuit model with thermal resistance correction," in The Journal of Engineering, 2019, vol. 2019, no. 16, pp. 2036-2041.

[7] L. Brabetz, M. Ayeb and H. Neumeier, "A New Approach to the Thermal Analysis of Electrical Distribution Systems," in SAE 2011 World Congress \& Exhibition, Detroit, MI, 2011.

[8] R. S. Olsen, J. Holboll and U. S. Gudmundsdóttir, "Dynamic Temperature Estimation and Real Time Emergency Rating of Transmission Cables," in IEEE Power and Energy Society General Meeting, San Diego, CA, USA, 2012.

[9] S. Önal, M. Kiffmeier and S. Frei, "Modellbasierte intelligente Sicherungen mit umgebungsadaptiver Anpassung der Auslöseparameter," in International Conference EEHE, Electric \& Electronic in Hybrid and Electric Vehicles and Electrical Energy Management, Würzburg, Germany, 2018.

[10] F. Loos, K. Dvorsky and H.-D. Liess, "Two approaches for heat transfer simulation of current carrying multicables," in Mathematics and Computers in Simulation, 2014, vol. 101, pp. 13-30. 
[11] J. He, Y. Tang, B. Wei, J. Li, L. Ren, J. Shi, K. Wu, X. Li, Y. Xu and S. Wang, "Thermal Analysis of HTS Power Cable Using 3-D FEM Model," in IEEE Transactions on applied superconductivity, 2013, vol. 23, no.3.

[12] S. L. Rickman and C. J. Iannello, "Heat transfer analysis in wire bundles for aerospace vehicles," in International Conference on Simulation and Experiments in Heat Transfer and its Applications, 2016.

[13] C. R. Paul, Analysis of multiconductor transmission lines. 2. ed., Piscataway, NJ: IEEE Press, 2008.

[14] VDI-Gesellschaft Verfahrenstechnik und Chemieingenieurwesen, VDI-Wärmeatlas, Berlin: Springer, 2013.

[15] S. W. Churchill and H. H. Chu, "Correlating equations for laminar and turbulent free convection from a horizontal cylinder," in International Journal of Heat and Mass Transfer, 1975, vol. 18, no. 9, p. 1049-1053.

[16] J. Abate and W. Whitt, "A Unified Framework for Numerically Inverting Laplace Transforms," in INFORMS Journal on Computing, 2006, vol. 18, no. 4. 